in vivo $35: 3039-3044(2021)$

doi:10.21873/invivo.12599

Review

\title{
Drug Repositioning With an Anticancer Effect: Contributions to Reduced Cancer Incidence in Susceptible Individuals
}

\author{
SUNGPIL YOON and HYUNG SIK KIM \\ School of Pharmacy, Sungkyunkwan University, Suwon, Republic of Korea
}

\begin{abstract}
Certain diseases and age groups are associated with a higher incidence of cancer. Cancer prevention can be achieved using repositioned drugs that have anticancer ability, thereby reducing the incidence of cancer in susceptible individuals. This implies that the selection of repositioned drugs can have dual benefits: controlling preexisting diseases and facilitating cancer prevention. This report outlines the rationale underlying drug repositioning for medications with an anticancer effect and discusses its advantages. We discuss repositioned drugs with anticancer effects that may contribute to cancer prevention in susceptible individuals and the general population with temporary, treatable conditions. The discussion of drug repositioning in this review should facilitate the initiation of clinical trials and lead to therapeutic application of such drugs to reduce the incidence of cancer in susceptible individuals.
\end{abstract}

Repositioned drugs with anticancer effects may reduce cancer incidence in at-risk populations. For example, the incidence of cancer is higher in patients with diabetes than in individuals who do not have diabetes (1). Metformin, a well-known antidiabetic drug, has been associated with anticancer effects and can be considered as a repositioned drug (2). Several studies have shown that metformin has benefits over other drugs in reducing the incidence of cancer in patients with diabetes $(3,4)$,

This article is freely accessible online.

Correspondence to: Sungpil Yoon, Ph.D., School of Pharmacy, Sungkyunkwan University, 2066 Seobu-ro, Jangan-gu, Suwon, Gyeonggi-do, 16419, Republic of Korea. Tel: +82 1055024893, Fax: +82 312928800, e-mail: syoon88@gmail.com; Hyung Sik Kim, PhD School of Pharmacy, Sungkyunkwan University, 2066 Seobu-ro, Jangan-gu, Suwon, Gyeonggi-do, 16419, Republic of Korea. Tel: +82 312907789, Fax: +82 312928800, e-mail: hkims@skku.edu

Key Words: Drug repositioning, caner prevention, susceptible individuals, aging, metformin. implying that apart from its usage as an anti-diabetic drug, it can be used for new indications, in this case, as a drug for the prevention of cancer. Therefore, among the various medications used for diabetes, selective administration of metformin can maximize cancer prophylaxis in high-risk patients with diabetes.

Certain diseases and age groups are associated with a higher incidence of cancer. As seen with metformin, drug selection can reduce the incidence of cancer in susceptible patients, especially in those who take repositioned drugs that have cancer preventative effects. The use of repositioned drugs in these cancer-susceptible patients provides the dual benefits of treating disease and preventing cancer. Therefore, it is important, not only for patients, but also for pharmacists or doctors to enhance their knowledge about such repositioned drugs. It may also contribute to cancer prophylaxis in the general population treated temporarily with drugs for certain diseases, such as malaria and bacterial infections. Below is a summary of the various patient groups that could benefit from the repositioning of drugs with anticancer effects (Figure 1).

Patients with blood-related diseases. Owing to the increased occurrence of cancer in patients with blood-related diseases, greater attention is needed on cancer prevention. Aspirin is used to reduce pain, fever, or inflammation. It is also used for hypertension and as a long-term medication to help prevent further heart attacks and blood clots in high-risk individuals. Similar to metformin, population studies have shown that aspirin can reduce the incidence of cancer $(5,6)$. Statins (HMG-CoA reductase inhibitors) are used as lipidlowering medications or to control high cholesterol levels and have shown cancer-reducing effects $(7,8)$. Hence, the selection of drugs such as metformin, aspirin, or statins, which possess cancer-reducing abilities in addition to their original intended treatment effect, would allow the dual control of blood-related diseases and cancer.

Patients with human immunodeficiency virus (HIV). It has been observed that patients with HIV have a higher incidence of 


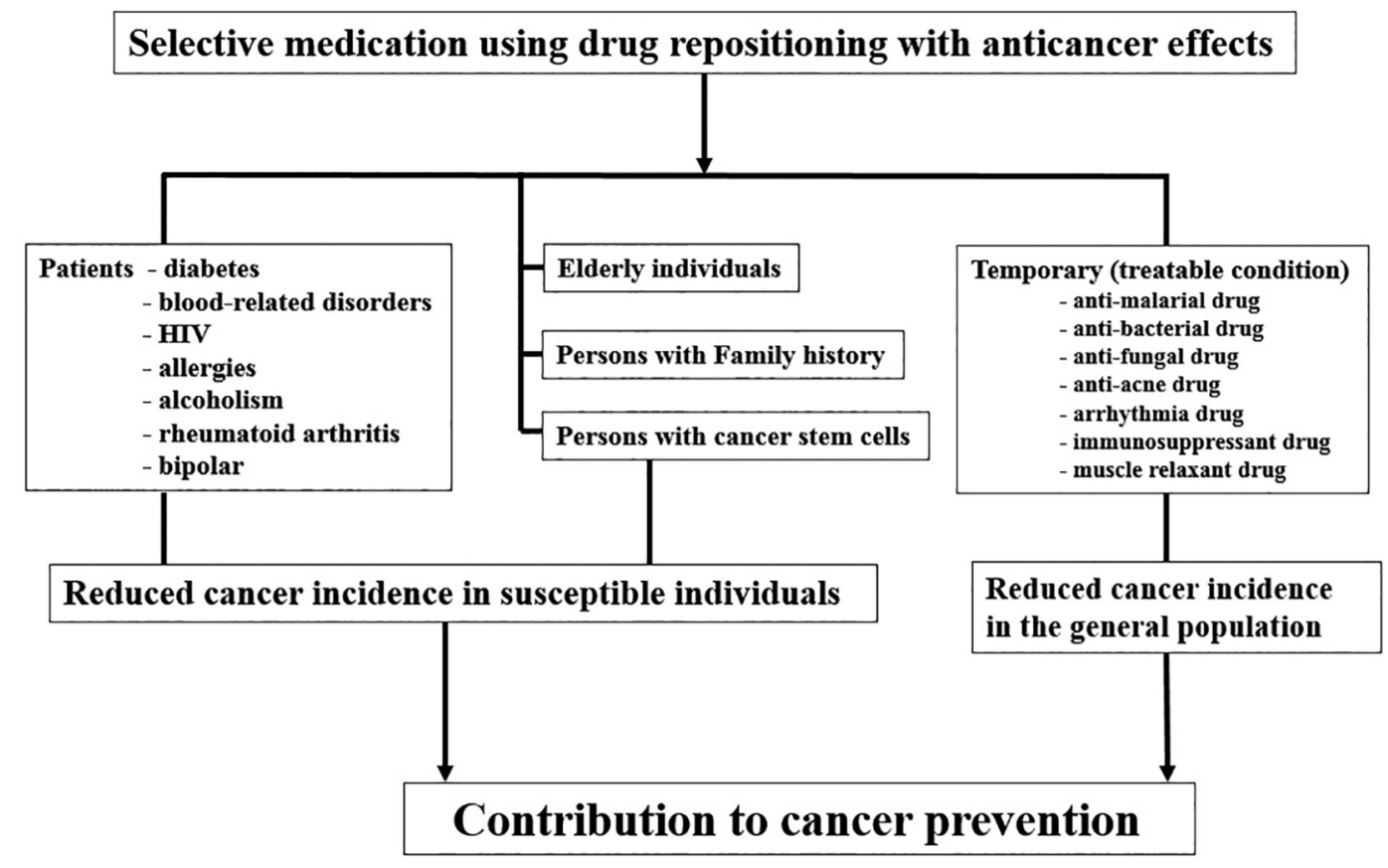

Figure 1. Summary of the various patient groups that could benefit from the repositioning of drugs with anticancer effects. These repositioned drugs can contribute to cancer prevention in cancer-susceptible individuals or in the general population. This implies that the selection of repositioned drugs can have dual benefits in controlling pre-existing diseases and facilitating cancer prevention.

cancer during their lifetime (9). Protease inhibitor-based drugs for HIV-infected cells have been developed, and these drugs (notably nelfinavir and lopinavir) have been shown to be effective in cancer treatment (10). Clinical trials with nelfinavir and lopinavir have been conducted in patients with cancer (11) and can be considered for drug repositioning. Other HIV drugs, such as atazanavir or ritonavir, have demonstrated sensitization of cancer cells; however, further investigation should facilitate the selection of a personalized drug treatment strategy for the regulation of HIV and the prevention of cancer.

Patients with allergies. The presence of allergies has also been correlated with a higher incidence of cancer, especially in older patients (12). Histamine receptor antagonists are generally used to control various forms of allergies (13). As many histamine receptor antagonists have been developed, patients with allergies may be treated with a wide variety of drugs. Some allergy drugs, such as loratadine or azelastine, have demonstrated anticancer effects and may be used as repositioned drugs (14). Selective and continuous medication with these anti-allergy drugs may contribute to a reduction in the incidence of cancer in patients with allergies, especially in elderly patients. Further studies on various histamine receptor antagonists with anticancer effects may facilitate the prescription of effective personalized medication for cancer prevention in patients with allergies.
Patients with alcoholism. Alcohol consumption is an important risk factor associated with cancer; as such, it is important to reduce or avoid alcohol consumption, particularly in patients with hepatitis virus or those with a family history of liver cancer. Although this is common knowledge, some individuals find it difficult to avoid alcohol consumption. The administration of drugs for alcohol abuse can help these individuals. One such drug is disulfiram, which has demonstrated both cancer-sensitizing and cancer-reducing abilities in patients with alcohol addiction $(15,16)$. Given that the prescription of disulfiram can aid cancer prevention in patients with alcoholism, earlier and continuous treatment with disulfiram may lower their risk of cancer.

Patients with rheumatoid arthritis. Rheumatoid arthritis is an auto-immune disease associated with increased inflammation (17). Inflammation-related diseases, such as rheumatoid arthritis, are also known to result in an increased incidence of cancer. Many drugs have been investigated and developed for regulating the immune system or inflammation. Celecoxib has been used as a drug for the treatment of rheumatoid arthritis. Many studies have demonstrated the usefulness of celecoxib as a repositioned drug for patients with cancer, owing to its anticancer effect (18). Hence, the use of this drug in patients with rheumatoid arthritis can help 
treat this disease, as well as reduce the incidence of cancer. Further population-based studies may facilitate the prescription of this medication for cancer prevention in patients with rheumatoid arthritis.

Elderly patients with various diseases. The incidence of cancer has been found to increase with age owing to the accumulation of DNA mutations over time and the increased formation of cancerous cells. Therefore, aging can be considered as an important risk factor for the development of cancer. The functionality of various organs and tissues is reduced as people age and medications are required to overcome these impairments. Examples of drugs used for the treatment of dysfunctional tissues or organs that also have anticancer effects are described below. This information can be advantageous in elderly patients, as apart from the treatment of diseases related to abnormal tissues or drugs, these drugs can also assist in cancer prevention. (A) Patients with osteoporosis have an increased risk of fractures. For post-menopausal women, the incidence of osteoporosis is very high. The most common medication used for the treatment of osteoporosis is zoledronic acid, which also has anticancer effects (19). (B) Elderly male patients with erectile dysfunction generally change their lifestyle (exercising or sleeping) or modify their diet to overcome the disorder. However, if these natural methods are not effective, they are treated with sildenafil. Studies have shown sildenafil to have anticancer effects in various cancer models $(20,21)$. (C) Elderly individuals have a higher risk of Alzheimer's disease. As the average human lifespan increases, Alzheimer's disease is emerging as a major health issue in the elderly population and effective medication is required for its treatment and prevention. Donepezil, a well-known drug used for the treatment of Alzheimer's disease, has demonstrated anticancer effects $(20,22)$, suggesting that the treatment of Alzheimer's disease with donepezil may also reduce the incidence of cancer in elderly patients.

Patients with schizophrenic disorders. Many anti-psychotic drugs used for the treatment of bipolar disorder or schizophrenia have been found to have anticancer effects and have been tested as repositioned drugs in patients with cancer. Most of these drugs target the dopamine or histamine receptors on the cell membrane to block the extracellular signals that are responsible for abnormal responses in the brain cells. Considering that mutations in the membranerelated receptors or kinases are correlated with cancer growth, it is assumed that drugs that target these cellular membrane receptors may also possess anticancer effects. Drugs used for the treatment of bipolar disorders that can be repositioned include fluphenazine, thioridazine, pimozide, aripiprazole, and valproic acid (23-27). As these drugs have anticancer effects, several clinical trials have been conducted to test their efficacy in various types of solid tumors, as well as in combination with other chemotherapeutic drug therapies. Further population-based studies are needed for enhanced cancer prevention in patients with bipolar disease.

Patients treated for short term with medications for various diseases. Many drugs used for the short-term treatment of various diseases have anticancer effects and may contribute to a lower incidence of cancer. (A) Anti-malarial drugs are often taken before traveling to countries that have a higher incidence of malarial infection. Patients who are already infected with malaria are administered anti-malarial drugs. Because of resistance to anti-malarial drugs, investigators are continuously developing novel drugs. Among these, mefloquine, primaquine, and chloroquine have shown anticancer effects $(28,29)$. These drugs have been tested in patients with cancer in various clinical trials. Chloroquine has been investigated widely owing to its specific mechanism of action in various cancer models. Chloroquine can sensitize cancer cells through the inhibition of autophagy and antiangiogenic activity $(30,31)$. (B) Various anti-bacterial drugs have demonstrated anticancer effects. For example, nitroxoline is used for the treatment of bacterial infections in the urinary tract and has shown anti-angiogenesis effects (32). (C) The anti-fungal drug, itraconazole, has also shown anticancer effects (33). (D) Medications have been developed for the treatment of acne in young individuals. Minocycline, an anti-acne drug, has been shown to exert anticancer effects via reduction in the levels of matrix metalloproteinases (34). Therefore, the administration of minocycline in individuals with acne may be beneficial in lowering cancer incidence. (E) Digoxin, a drug used for the treatment of arrhythmia or heart failure, has also shown anticancer effects (35). (F) Immunosuppressant drugs, such as mycophenolic acid and rapamycin, used for patients who have undergone organ transplantation surgery, have demonstrated anticancer effects (36). Notably, rapamycin has been found to be effective against various tumors and even against cancers resistant to chemotherapy, owing to its energy metabolism-targeting action. (G) Thiocolchicoside, a muscle relaxant drug with an anti-inflammatory effect, and riluzole, used in neuronal diseases, have shown cancer-sensitizing effects $(37,38)$. More investigations are needed to categorize and investigate these repositioned drugs and inform the patients accordingly regarding their further usefulness in cancer prevention.

Individuals with potential cancer stem cells. The repositioning of drugs with anticancer effects can be subdivided based on their usefulness in cancers with multidrug resistance (MDR) phenotypes. Frequent exposure to anticancer drugs leads to the development of MDR in cancer cells $(39,40)$. The overexpression of P-glycoprotein (P-gp) is one of the common mechanisms of resistance to anticancer 
drugs $(41,42)$. Therefore, investigators have studied novel therapeutic options that target $\mathrm{P}$-gp-over-expressing resistant cancer cells. Among the repositioned drugs described earlier, aripiprazole, nelfinavir, and azelastine have shown high $\mathrm{P}$ gp inhibitory activities and thus can be used to sensitize Pgp-over-expressing resistant cancers $(41,43)$. Reserpine, a hypertension drug, is also known to have significant P-gp inhibitory effects on MDR cancer cells (44). Drugs that have been subdivided based on specific anticancer effects, such as P-gp inhibitory activity, can be prescribed as repositioned drugs in cancer-susceptible individuals. Considering that cancer stem cells develop throughout an individual's lifetime, repositioned drugs with MDR-targeting ability are assumed to have higher chances of reducing cancer incidence.

Conclusion and further studies. Cancer prevention can be achieved using repositioned drugs that have anticancer ability, thereby reducing the incidence of cancer in susceptible individuals. This implies that the repositioned drugs have dual benefits in controlling pre-existing diseases and facilitating cancer prevention. As aging and family history are strong risk factors associated with cancer, the use of repositioned drugs has been suggested in older patients who have a family history of cancer.

With the advent and growth of personalized medicine, there has been a surge in the number of drugs developed for various diseases. Further research is warranted to investigate whether these drugs have anticancer effects and to examine if they can be suitable for drug repositioning. For example, benzodiazepines, alprazolam, clonazepam, chlordiazepoxide, and diazepam (45), which are used for the treatment of anxiety disorders, can be investigated for anticancer effects, their mechanism of action, and their specific tumor-targeting properties. It is also important to consider that long-term treatment with certain drugs can increase the incidence of cancer and should be avoided in cancer-susceptible individuals.

In addition, clinical databases for population-based drug treatments should be well-organized, analyzed, and continuously updated. This includes correlations between period or type of drug administration and cancer incidence in various populations. It is hypothesized that such information could contribute to the prevention of cancer by reducing the incidence of cancer in susceptible individuals.

\section{Conflicts of Interest}

The Authors declare no conflicts of interest regarding this study.

\section{Authors' Contributions}

Sungpil Yoon and Hyung Sik Kim: Conceived, designed, and wrote the article. All Authors read and approved the final manuscript.

\section{Acknowledgements}

This work was supported by National Research Foundation of Korea (NRF) funded by the Ministry of Education (NRF2019R1A2C2002923).

\section{References}

1 Suh S and Kim KW: Diabetes and Cancer: Cancer should be screened in routine diabetes assessment. Diabetes Metab J 43(6): 733-743, 2019. PMID: 31902143. DOI: 10.4093/dmj.2019.0177

2 Yoon S, Wang X, Vongpunsawad S, Tromp G and Kuivaniemi H: Editorial: FDA-approved drug repositioning for P-glycoprotein overexpressing resistant cancer. Front Oncol 11: 632657, 2021. PMID: 33816271. DOI: 10.3389/fonc.2021.632657

3 Campbell JM, Bellman SM, Stephenson MD and Lisy K: Metformin reduces all-cause mortality and diseases of ageing independent of its effect on diabetes control: A systematic review and meta-analysis. Ageing Res Rev 40: 31-44, 2017. PMID: 28802803. DOI: 10.1016/j.arr.2017.08.003

4 Schulten HJ: Pleiotropic effects of metformin on cancer. Int $\mathbf{J}$ Mol Sci 19(10): 2850, 2018. PMID: 30241339. DOI: 10.3390/ ijms 19102850

5 Drew DA, Cao Y and Chan AT: Aspirin and colorectal cancer: the promise of precision chemoprevention. Nat Rev Cancer 16(3): 173-186, 2016. PMID: 26868177. DOI: 10.1038/nrc.2016.4

6 Zhang X, Feng Y, Liu X, Ma J, Li Y, Wang T and Li X: Beyond a chemopreventive reagent, aspirin is a master regulator of the hallmarks of cancer. J Cancer Res Clin Oncol 145(6): 13871403, 2019. PMID: 31037399. DOI: 10.1007/s00432-01902902-6

7 Beckwitt $\mathrm{CH}$, Brufsky A, Oltvai $\mathrm{ZN}$ and Wells A: Statin drugs to reduce breast cancer recurrence and mortality. Breast Cancer Res 2O(1): 144, 2018. PMID: 30458856. DOI: 10.1186/s13058018-1066-z

8 Sopková J, Vidomanová E, Strnádel J, Škovierová H and Halašová $E$ : The role of statins as therapeutic agents in cancer. Gen Physiol Biophys 36(5): 501-511, 2017. PMID: 29372683. DOI: $10.4149 / \mathrm{gpb} \_2017045$

9 Gobert A, Veyri M, Poizot-Martin I, Lavolé A, Solas C, Paliche R, Katlama C, Costagliola D and Spano JP: [HIV and cancer: What's new in 2017?]. Bull Cancer 105(3): 256-262, 2018. PMID: 29548534. DOI: 10.1016/j.bulcan.2018.02.002

10 Kim JY, Park YJ, Lee BM and Yoon S: Co-treatment with HIV protease inhibitor nelfinavir greatly increases late-phase apoptosis of drug-resistant KBV20C cancer cells independently of P-glycoprotein inhibition. Anticancer Res 39(7): 3757-3765, 2019. PMID: 31262902 . DOI: 10.21873/anticanres.13524

11 Chow WA, Jiang C and Guan M: Anti-HIV drugs for cancer therapeutics: back to the future? Lancet Oncol 10(1): 61-71, 2009. PMID: 19111246. DOI: 10.1016/S1470-2045(08)70334-6

12 D'Arcy M, Rivera DR, Grothen A and Engels EA: Allergies and the subsequent risk of cancer among elderly adults in the United States. Cancer Epidemiol Biomarkers Prev 28(4): 741-750, 2019. PMID: 30700443. DOI: 10.1158/1055-9965.EPI-18-0887

13 Bachert C: A review of the efficacy of desloratadine, fexofenadine, and levocetirizine in the treatment of nasal congestion in patients with allergic rhinitis. Clin Ther 31(5): 921-944, 2009. PMID: 19539095. DOI: 10.1016/j.clinthera. 2009.05.017 
14 Kim JY, Kim KS, Kim IS and Yoon S: Histamine receptor antagonists, loratadine and azelastine, sensitize P-gpoverexpressing antimitotic drug-resistant KBV20C cells through different molecular mechanisms. Anticancer Res 39(7): 37673775, 2019. PMID: 31262903. DOI: 10.21873/anticanres.13525

15 Skrott Z, Mistrik M, Andersen KK, Friis S, Majera D, Gursky J, Ozdian T, Bartkova J, Turi Z, Moudry P, Kraus M, Michalova M, Vaclavkova J, Dzubak P, Vrobel I, Pouckova P, Sedlacek J, Miklovicova A, Kutt A, Li J, Mattova J, Driessen C, Dou QP, Olsen J, Hajduch M, Cvek B, Deshaies RJ and Bartek J: Alcohol-abuse drug disulfiram targets cancer via $\mathrm{p} 97$ segregase adaptor NPL4. Nature 552(7684): 194-199, 2017. PMID: 29211715. DOI: 10.1038/nature25016

16 Viola-Rhenals M, Patel KR, Jaimes-Santamaria L, Wu G, Liu J and Dou QP: Recent advances in antabuse (Disulfiram): The importance of its metal-binding ability to its anticancer activity. Curr Med Chem 25(4): 506-524, 2018. PMID: 29065820. DOI: $10.2174 / 0929867324666171023161121$

17 de Germay S, Bagheri H, Despas F, Rousseau V and Montastruc F: Abatacept in rheumatoid arthritis and the risk of cancer: a world observational post-marketing study. Rheumatology (Oxford) 59(9): 2360-2367, 2020. PMID: 31880308. DOI: 10.1093/rheumatology/kez604

18 Lim JS, Park Y, Lee BM, Kim HS and Yoon S: Co-treatment with celecoxib or NS398 strongly sensitizes resistant cancer cells to antimitotic drugs independent of P-gp inhibition. Anticancer Res 36(10): 5063-5070, 2016. PMID: 27798865. DOI: 10.21873/anticanres.11075

19 Hortobagyi GN, Van Poznak C, Harker WG, Gradishar WJ, Chew H, Dakhil SR, Haley BB, Sauter N, Mohanlal R, Zheng M and Lipton A: Continued treatment effect of zoledronic acid dosing every $12 v s .4$ weeks in women with breast cancer metastatic to bone: The OPTIMIZE-2 randomized clinical trial. JAMA Oncol 3(7): 906-912, 2017. PMID: 28125763. DOI: 10.1001/jamaoncol.2016.6316

20 Kim JY, Son JY, Lee BM, Kim HS and Yoon S: Aging-related repositioned drugs, donepezil and sildenafil citrate, increase apoptosis of anti-mitotic drug-resistant KBV20C cells through different molecular mechanisms. Anticancer Res 38(9): 51495157, 2018. PMID: 30194162. DOI: 10.21873/anticanres.12837

21 El-Naa MM, Othman M and Younes S: Sildenafil potentiates the antitumor activity of cisplatin by induction of apoptosis and inhibition of proliferation and angiogenesis. Drug Des Devel Ther 10: 3661-3672, 2016. PMID: 27895461. DOI: 10.2147/DDDT.S107490

22 Rapp SR, Case LD, Peiffer A, Naughton MM, Chan MD, Stieber VW, Moore DF Jr, Falchuk SC, Piephoff JV, Edenfield WJ, Giguere JK, Loghin ME and Shaw EG: Donepezil for irradiated brain tumor survivors: A Phase III randomized placebocontrolled clinical trial. J Clin Oncol 33(15): 1653-1659, 2015. PMID: 25897156. DOI: 10.1200/JCO.2014.58.4508

23 Cheon JH, Lee BM, Kim HS and Yoon S: Highly halavenresistant KBV20C cancer cells can be sensitized by co-treatment with fluphenazine. Anticancer Res 36(11): 5867-5874, 2016. PMID: 27793910. DOI: 10.21873/anticanres.11172

24 Jiang C, Lee SH, Park JH, Lee JS, Park JW, Kim JR, Lee SH, Kim HS and Yoon S: A low dose of aripiprazole has the strongest sensitization effect among 19 repositioned bipolar drugs in P-gp-overexpressing drug-resistant cancer cells. Anticancer Res 41(2): 687-697, 2021. PMID: 33517273. DOI: 10.21873 /anticanres. 14820
25 Kim JY, Park Y, Lee BM, Kim HS and Yoon S: P-gp inhibition by the anti-psychotic drug pimozide increases apoptosis, as well as expression of $\mathrm{pRb}$ and $\mathrm{pH} 2 \mathrm{AX}$ in highly drug-resistant KBV20C cells. Anticancer Res 38(10): 5685-5692, 2018. PMID: 30275188. DOI: 10.21873 /anticanres. 12905

26 Kim JY, Tae IH, Lee BM, Kim HS and Yoon S: Low doses of the anti-psychotic drug aripiprazole have strong P-gp-inhibitory activity and sensitize anti-mitotic drug-resistant cancer cells. Anticancer Res 38(9): 5101-5108, 2018. PMID: 30194155. DOI: 10.21873/anticanres. 12830

27 Heers H, Stanislaw J, Harrelson J and Lee MW: Valproic acid as an adjunctive therapeutic agent for the treatment of breast cancer. Eur J Pharmacol 835: 61-74, 2018. PMID: 30075223. DOI: 10.1016/j.ejphar.2018.07.057

28 Choi AR, Kim JH, Cheon JH, Kim HS and Yoon S: Attenuation of colchicine toxicity in drug-resistant cancer cells by cotreatment with anti-malarial drugs. Anticancer Res 36(11): 58595866, 2016. PMID: 27793909. DOI: 10.21873/anticanres.11171

29 Choi AR, Kim JH, Woo YH, Kim HS and Yoon S: Anti-malarial drugs primaquine and chloroquine have different sensitization effects with anti-mitotic drugs in resistant cancer cells. Anticancer Res 36(4): 1641-1648, 2016. PMID: 27069141.

30 Maes H, Kuchnio A, Peric A, Moens S, Nys K, De Bock K, Quaegebeur A, Schoors S, Georgiadou M, Wouters J, Vinckier S, Vankelecom H, Garmyn M, Vion AC, Radtke F, Boulanger C, Gerhardt H, Dejana E, Dewerchin M, Ghesquière B, Annaert W, Agostinis $\mathrm{P}$ and Carmeliet P: Tumor vessel normalization by chloroquine independent of autophagy. Cancer Cell 26(2): 190206, 2014. PMID: 25117709. DOI: 10.1016/j.ccr.2014.06.025

$31 \mathrm{Xu} \mathrm{R}$, Ji Z, Xu C and Zhu J: The clinical value of using chloroquine or hydroxychloroquine as autophagy inhibitors in the treatment of cancers: A systematic review and meta-analysis. Medicine (Baltimore) 97(46): e12912, 2018. PMID: 30431566. DOI: $10.1097 / M D .0000000000012912$

32 Veschi S, De Lellis L, Florio R, Lanuti P, Massucci A, Tinari N, De Tursi M, di Sebastiano P, Marchisio M, Natoli C and Cama A: Effects of repurposed drug candidates nitroxoline and nelfinavir as single agents or in combination with erlotinib in pancreatic cancer cells. J Exp Clin Cancer Res 37(1): 236, 2018. PMID: 30241558. DOI: 10.1186/s13046-018-0904-2

33 Lee WH, Loo CY, Ghadiri M, Leong CR, Young PM and Traini D: The potential to treat lung cancer via inhalation of repurposed drugs. Adv Drug Deliv Rev 133: 107-130, 2018. PMID: 30189271. DOI: 10.1016/j.addr.2018.08.012

34 Zhou YQ, Liu DQ, Chen SP, Sun J, Wang XM, Tian YK, Wu W and Ye DW: Minocycline as a promising therapeutic strategy for chronic pain. Pharmacol Res 134: 305-310, 2018. PMID: 30042091. DOI: 10.1016/j.phrs.2018.07.002

35 Karasneh RA, Murray LJ, Hughes CM and Cardwell CR: Digoxin use after diagnosis of prostate cancer and survival: a populationbased cohort study. Pharmacoepidemiol Drug Saf 25(9): 10991103, 2016. PMID: 27146482. DOI: 10.1002/pds.4018

36 Geissler EK: Can immunosuppressive strategies be used to reduce cancer risk in renal transplant patients? Transplant Proc 42(9 Suppl): S32-S35, 2010. PMID: 21095449. DOI: 10.1016/ j.transproceed 2010.07 .004

37 Reuter S, Gupta SC, Phromnoi K and Aggarwal BB: Thiocolchicoside suppresses osteoclastogenesis induced by RANKL and cancer cells through inhibition of inflammatory pathways: a new use for an old drug. Br J Pharmacol 165(7): 
2127-2139, 2012. PMID: 21955206. DOI: 10.1111/j.14765381.2011.01702.x

38 Yu LJ, Wall BA and Chen S: The current management of brain metastasis in melanoma: a focus on riluzole. Expert Rev Neurother 15(7): 779-792, 2015. PMID: 26092602. DOI: 10.1586/14737175.2015.1055321

39 Wu Q, Yang Z, Nie Y, Shi Y and Fan D: Multi-drug resistance in cancer chemotherapeutics: mechanisms and lab approaches. Cancer Lett 347(2): 159-166, 2014. PMID: 24657660. DOI: 10.1016/j.canlet.2014.03.013

40 Zhang Q, Feng Y and Kennedy D: Multidrug-resistant cancer cells and cancer stem cells hijack cellular systems to circumvent systemic therapies, can natural products reverse this? Cell Mol Life Sci 74(5): 777-801, 2017. PMID: 27622244. DOI: 10.1007/ s00018-016-2362-3

41 Chen Z, Shi T, Zhang L, Zhu P, Deng M, Huang C, Hu T, Jiang $\mathrm{L}$ and Li J: Mammalian drug efflux transporters of the ATP binding cassette $(\mathrm{ABC})$ family in multidrug resistance: A review of the past decade. Cancer Lett 370(1): 153-164, 2016. PMID: 26499806. DOI: 10.1016/j.canlet.2015.10.010

42 Kim KS, Jiang C, Kim JY, Park JH, Kim HR, Lee SH, Kim HS and Yoon S: Low-dose crizotinib, a tyrosine kinase inhibitor, highly and specifically sensitizes P-glycoprotein-overexpressing chemoresistant cancer cells through induction of late apoptosis in vivo and in vitro. Front Oncol 10: 696, 2020. PMID: 32528877. DOI: $10.3389 /$ fonc. 2020.00696
43 Shukla S, Wu CP and Ambudkar SV: Development of inhibitors of ATP-binding cassette drug transporters: present status and challenges. Expert Opin Drug Metab Toxicol 4(2): 205-223, 2008. PMID: 18248313. DOI: 10.1517/17425255.4.2.205

44 Figueroa-González G, Jacobo-Herrera N, Zentella-Dehesa A and Pereda-Miranda R: Reversal of multidrug resistance by morning glory resin glycosides in human breast cancer cells. J Nat Prod 75(1): 93-97, 2012. PMID: 22148475. DOI: 10.1021/np200864m

45 Altamura AC, Moliterno D, Paletta S, Maffini M, Mauri MC and Bareggi S: Understanding the pharmacokinetics of anxiolytic drugs. Expert Opin Drug Metab Toxicol 9(4): 423-440, 2013. PMID: 23330992. DOI: 10.1517/17425255.2013.759209
Received July 8, 2021

Revised July 29, 2021

Accepted July 30, 2021 